\title{
Mechanisms and advancement of antifading agents for fluorescence microscopy and single-molecule spectroscopy $\dagger \ddagger$
}

\author{
Thorben Cordes, ${ }^{* a b}$ Andreas Maiser ${ }^{c}$ Christian Steinhauer, ${ }^{a}$ Lothar Schermelleh $* c$ \\ and Philip Tinnefeld $* a d$
}

Received 23rd September 2010, Accepted 14th January 2011

DOI: $10.1039 /$ c0cp01919d

Modern fluorescence microscopy applications go along with increasing demands for the employed fluorescent dyes. In this work, we compared antifading formulae utilizing a recently developed reducing and oxidizing system (ROXS) with commercial antifading agents. To systematically test fluorophore performance in fluorescence imaging of biological samples, we carried out photobleaching experiments using fixed cells labeled with various commonly used organic dyes, such as Alexa 488, Alexa 594, Alexa 647, Cy3B, ATTO 550, and ATTO 647N. Quantitative evaluation of (i) photostability, (ii) brightness, and (iii) storage stability of fluorophores in samples mounted in different antifades (AFs) reveal optimal combinations of dyes and AFs. Based on these results we provide guidance on which AF should preferably be used with a specific dye. Finally, we studied the antifading mechanisms of the commercial AFs using single-molecule spectroscopy and reveal that these empirically selected AFs exhibit similar properties to

ROXS AFs.

\section{Introduction}

Fluorescence light microscopy has become an indispensable tool in various scientific fields, ranging from biomedical research to material sciences. One of its key features is the possibility to specifically label and detect structural components of interest with spectrally distinct fluorophores, e.g., to analyze the spatial distribution of biomolecules within cells and tissues by immunohistochemistry. ${ }^{1}$ More recently, the introduction of single-molecule approaches ${ }^{2,3}$ and super-resolution imaging techniques, ${ }^{4-7}$ have further extended the capabilities and range of applications. Modern fluorescence applications are strongly

${ }^{a}$ Applied Physics - Biophysics \& Center for NanoScience (CeNS), Ludwig Maximilian University of Munich, Amalienstr. 54, 80799 Munich,Germany.E-mail: thorben.cordes@physik.lmu.de, p.tinnefeld@tu-braunschweig.de; Fax: +495313915334

${ }^{b}$ Biological Physics Research Group, Department of Physics,

University of Oxford, Clarendon Laboratory, Parks Road,

Oxford OX1 3PU, United Kingdom

${ }^{c}$ LMU Biocenter, Department of Biology, Ludwig Maximilian

University of Munich, Grosshaderner Str. 2,

82152 Planegg-Martinsried, Germany.

E-mail: lothar.schermelleh@Imu.de

${ }^{d}$ NanoBioSciences, Institute of Physical and Theoretical Chemistry, TU Braunschweig, Hans-Sommer-Str. 10, 38106 Braunschweig, Germany

$\dagger$ This article was submitted as part of a Themed Issue on singlemolecule optical studies of soft and complex matter. Other papers on this topic can be found in issue 5 of vol. 13 (2011). This issue can be found from the PCCP homepage http://www.rsc.org/pccp.

$\ddagger$ Electronic supplementary information (ESI) available. See DOI: $10.1039 / \mathrm{c} 0 \mathrm{cp} 01919 \mathrm{~d}$ dependent on the performance and characteristics of the fluorescent probes used. Important properties of the labels are their brightness (given by the product of extinction coefficient at the excitation wavelength and the fluorescence quantum yield), photostability (i.e., resistance to irreversible, light-induced reactions), storage stability of stained samples, solubility in water (for biological applications), the capability to chemically link the label to the structure of interest and finally a minimized influence of the label onto the labeled structure itself. Organic fluorophores often represent the premier choice due to their brightness, chemical flexibility, small size and many new labeling strategies even for in vivo applications. ${ }^{1,8}$

Many substances, especially reductants such as ascorbic acid (AA), 6-hydroxy-2,5,7,8-tetramethylchroman-2-carboxylic acid (Trolox, TX), ${ }^{9,10} p$-phenylenediamine (PPD, used in the commercial product Vectashield), 1,4-diazabicyclo[2.2.2]octane $\left(\mathrm{DABCO}\right.$, used in Ibidi-MM) ${ }^{11}$ or n-propyl gallate and triplet-quenchers such as mercaptoethylamine ${ }^{12}$ and cyclooctatetraene, ${ }^{12,13}$ have been known to improve the photostability of fluorophores for microscopy and single-molecule spectroscopy. ${ }^{14,15}$ Antifading substances are typically dissolved in glycerol-buffer or aqueous solution, which preserve the sample morphology. It should be noted that other frequently used embedding media have polymerizing formulae (e.g., Prolong Gold, Moviol). While good antifading properties have been reported, ${ }^{14,15}$ cells embedded in these hardening media show substantial flattening, which is often disadvantageous for applications where the preservation of the 3-dimensional (3D) morphology is a requirement. Herein, we compare a 


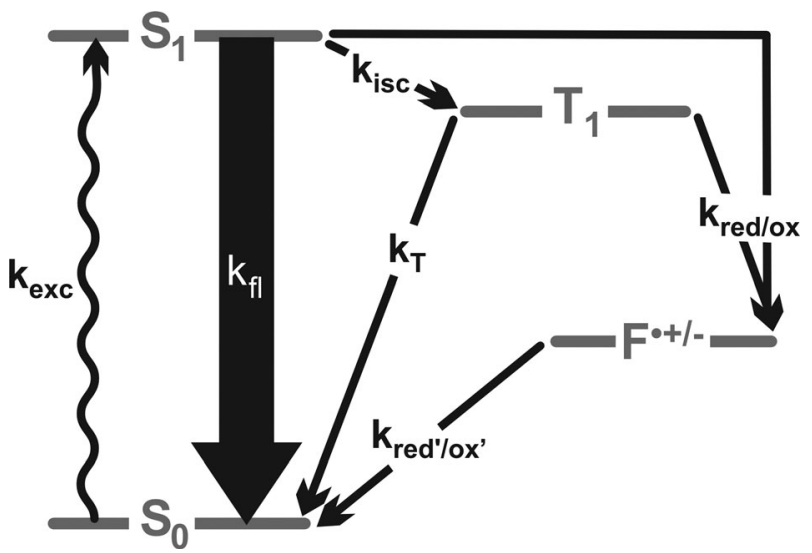

Fig. 1 Jablonski diagram according to the ROXS concept: A single fluorophore is cycled between its singlet ground state $\left(S_{0}\right)$ and the fluorescent first excited state $\left(\mathrm{S}_{1}\right)$ with the excitation rate $k_{\text {exc }}$ emitting a characteristic number of photons. The probability to enter the dark triplet state $\left(\mathrm{T}_{1}\right)$ with its inverse lifetime $k_{\mathrm{T}}$ is given by the rate constant of intersystem crossing $\left(k_{\text {isc }}\right)$. As the high reactivity of the triplet state can cause irreversible destruction of the fluorophore it is rapidly depleted with rates $k_{\text {red }}$ /ox by oxidizing or reducing agents forming a radical cation or anion $\left(\mathrm{F}^{\bullet \pm}\right)$, respectively. Each of the ionized states is depleted by the complementary redox reaction with rate constants $k_{\mathrm{red}^{\prime} / \mathrm{ox}^{\prime}}$ recovering the electronic ground state.

recently developed antifading formula that simultaneously employs reducing and oxidizing agents ${ }^{16}$ with common, commercial, glycerol-based antifading formulae suited for (3D) fluorescence microscopy. ${ }^{11,14,15}$

In contrast to the empirically found AFs, a recently established formula using a reducing and oxidizing system (ROXS) is based on a photophysical model. The underlying rationale is that an antifading formula has to rapidly and effectively depopulate reactive intermediate states other than the singlet states $S_{0}$ and $S_{1}$ that are part of the fluorescence excitation-emission cycle. Every time the fluorophore enters a potentially reactive state of a lifetime significantly longer than $S_{1}$ it should be depopulated to ultimately restore the ground state (see Fig. 1 for energy level diagram).

This should not only increase photostability but dependent on the excitation conditions also increase the brightness of the fluorophore. The ROXS concept realizes this rationale using electron transfer reactions. As soon as a transient state of higher energy - which is a potential reactive intermediate on common photobleaching pathways such as the triplet state-is formed, it is depopulated through an electron transfer reaction either with the reductant or with the oxidant. In the case of a reduction, a radical anion is formed whereas in the case of a primary reaction with an oxidant the corresponding radical cation is formed. In the following, these transient states are also rapidly depopulated through the complementary electron transfer reaction, that is, the radical anion will react with the oxidant to form the ground state. Alternatively, the radical cation reacts with the reductant also yielding the ground state. In all instances, the ground state is rapidly formed and the build-up of a broad range of transient states including triplet and radical states is suppressed (see scheme in Fig. 1). These reactions are commonly diffusion controlled.

The ROXS concept was demonstrated to efficiently eliminate blinking (due to triplet states as well as redox blinking due to radical ion states) from single-molecule intensity transients for a variety of different fluorophore classes (cyanines, (carbo-) rhodamines, oxazines) by different combinations of oxidants and reductants. ${ }^{10,16-18}$ For most fluorophores, particularly in the yellow and red spectral regions a drastic increase of photostability was observed. ${ }^{18}$ Targeted induction of transient dark states by adapting the concentrations of redox active agents was also exploited for super-resolution imaging by different approaches. ${ }^{19-21}$ But the implementation of ROXS in common biological imaging, e.g., confocal microscopy,
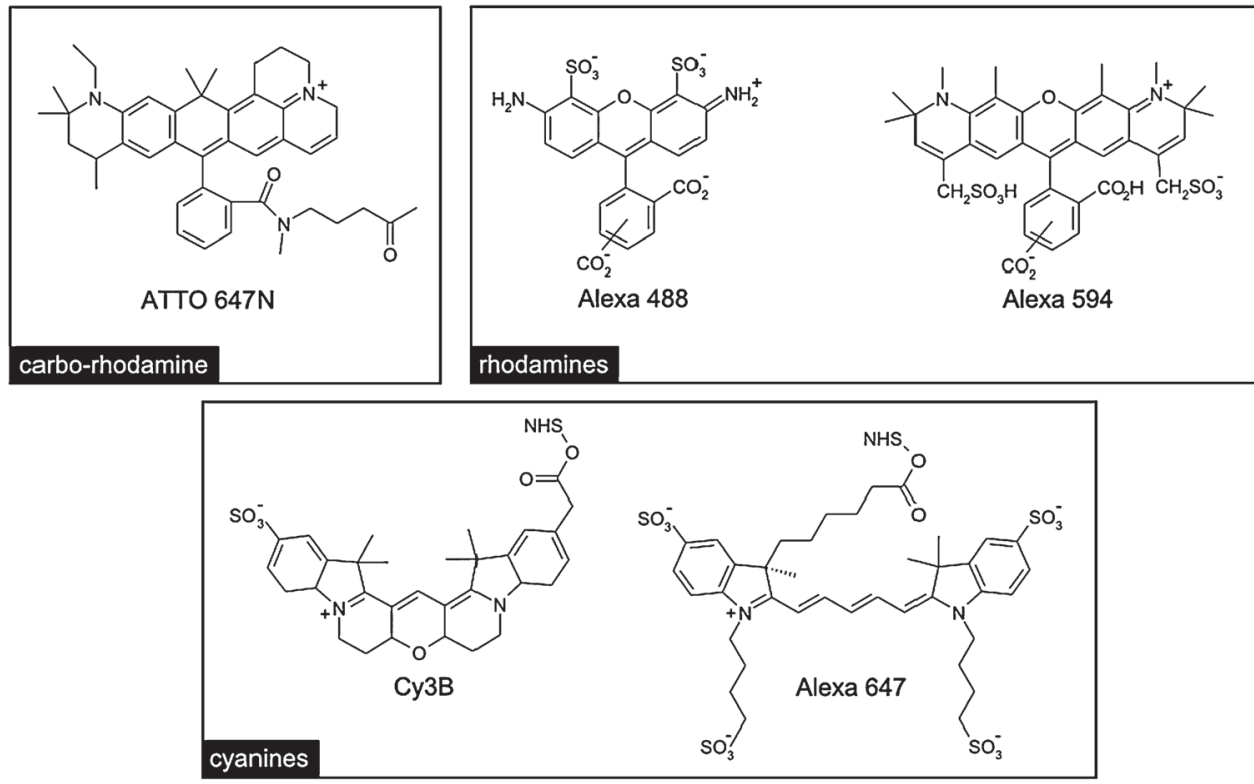

Scheme 1 Molecular structures of fluorophores investigated in the present study: ATTO $647 \mathrm{~N},{ }^{22}$ Alexa $488 / 594,{ }^{23} \mathrm{Cy} 3 \mathrm{~B},{ }^{24}$ Alexa $647 .{ }^{25}$ The dyes are sorted according to the class of fluorophores (carbo-rhodamines, rhodamines, cyanines). The structure of ATTO 550 was not available. 
and a comparison with common AFs used for immunofluorescence is still missing.

In this paper, we compare the performance of two ROXS AFs (ascorbic acid/methyl viologen, Trolox/Trolox-quinone) with common substances also used in commercial AFs such as phenylenediamine and DABCO. We therefore carried out spinning disk confocal microscopy in combination with repetitive point laser scanning for the imaging and controlled bleaching of immunolabeled nuclear proteins in fixed mammalian tissue culture cells. From these data sets we extracted the parameters (i) photostability and (ii) brightness. By measuring the same samples again after three days of storage we additionally obtained the (iii) storage stability in the respective mounting medium. To test for the general validity of our results we characterized various organic dyes of different dye classes, such as cyanines and (carbo)-rhodamines, spanning the entire visible spectrum. For all studied dyes (Alexa 488, Alexa 594, Alexa 647, Cy3B, ATTO 550, ATTO 647N), available molecular structures are shown in Scheme 1.

Our data suggest that the ROXS based AFs significantly improve the performance of dyes in immunofluorescence imaging and often outperform classical AFs. We, however, also find more specific effects that we summarize in practical guidance outlining which particular AF should be used for a given fluorophore (class).

To learn more about the mechanism of the empirically found antifading agents we finally used single-molecule spectroscopy and studied the influence of different AF concentrations on the blinking of single dye molecules. Therefore, we used the electron affine oxazine ATTO 655 that has been termed single-molecule redox sensor (SMRS) because it selectively reports on the oxidizing and reducing properties of the environment: the lifetime of the dark state reports on the oxidizing properties of the medium whereas the number of emitted photons for one $\mathrm{ON}$-state reports on the reducing properties of the medium. ${ }^{10}$ Shortening OFF-times at constant emitted photons of the SMRS have recently revealed the antifading mechanism of Trolox (TX) that forms a quinone over time and then acts according to the ROXS concept. $^{10}$

For all commercial AFs we found shortening OFF-state lifetimes at increasing AF-concentration indicating unexpected and substantial oxidizing capabilities of the AFs that are considered to be reductants. This leads to the conclusion that their empirical selection might be related to their additional oxidizing properties and that their stabilizing mechanism is also related to the ROXS concept. This fact of course underlines the importance of the ROXS concept not only for singlemolecule spectroscopy but also for fluorescence applications in general.

\section{Material and methods}

\section{Immunostaining, confocal microscopy and photobleaching experiments}

Human HeLa cells stably expressing histone H2B-GFP, and HeLa Kyoto cells were cultured in DMEM supplemented with $10 \%$ fetal bovine serum and $50 \mu \mathrm{g} \mathrm{ml}^{-1}$ gentamycine.
For photostability experiments cells were grown to $50-80 \%$ confluency on $18 \times 18 \mathrm{~mm}$ coverslips before fixation with $3.7 \%$ formaldehyde (Sigma) in phosphate buffered saline (PBS). All washing steps after fixation were performed with $0.02 \%$ Tween in PBS (PBST). Cells were permeabilized with $0.5 \%$ Triton X-100 in PBS. GFP-fusion proteins were stained with GFP-binding camelide-derived single chain antibody fragments $^{26}$ (GFP-binding protein; GBP) conjugated to Cy3b, ATTO 550 or ATTO 647N (ChromoTek) diluted in blocking solution ( $2 \%$ BSA/PBST). Alternatively, HeLa cells were stained with primary mouse monoclonal antibodies against Pan-histone (Roche) and secondary anti-mouse antibodies labeled with Alexa 488, Alexa 594, Alexa 647 (Invitrogen). Cells were counterstained with $100 \mathrm{ng} \mathrm{ml}^{-1}$ 4',6-diamidino-2- $^{\prime}$ indole (DAPI) in PBST for $5 \mathrm{~min}$ and mounted on microscope slides with indicated mounting media. In the case of the ROXS-buffers oxygen was removed from the sample using an oxygen-scavenging system based on glucose-oxidase/ catalase. ${ }^{27,28}$ The coverslip was sealed by mounting on a thin strip of silicone paste applied on the microscope slide filled with freshly prepared buffers or mounting media: (i) standard ROXS-buffer with $1 \mathrm{mM}$ concentrations of ascorbic acid (AA) and $1 \mathrm{mM} N, N^{\prime}$-methyl viologen (MV); ${ }^{16}$ (ii) ROXS-buffer containing $1 \mathrm{mM}$ Trolox (TX) that was additionally illuminated with UV-light (lamp-filter) for $10 \mathrm{~min}$ in order to form Troloxquinone (TQ) in sufficient concentrations $>25 \mu \mathrm{M} ;{ }^{9,10}$ (iii) pure Vectashield (VS) medium (Vector Labs); ${ }^{14}$ (iv) Ibidi-MM mounting-medium (Ibidi).

Photobleaching was performed with an UltraVIEW VoX spinning disc microscope equipped with an integrated FRAP PhotoKinesis accessory (PerkinElmer), assembled to an Axio Observer D1 inverted stand (Carl Zeiss) and using an oil immersion objective $(63 \times$, NA 1.40, PlanApo, Carl Zeiss). Confocal time series were recorded with intervals of 1 frame/s, exposure times of 100-200 ms, frame size of $1024 \times 1024$ pixels and 14-bit image depth. For each experiment the entire field of view containing approximately 5 to 20 cell nuclei was selected for bleaching. Depending on the tested fluorophore either one of the $488 \mathrm{~nm}, 561 \mathrm{~nm}$ or $635 \mathrm{~nm}$ laser line (measured laser power at the objective: $4.5 \mathrm{~mW}, 2,1 \mathrm{~mW}$ and $1,1 \mathrm{~mW}$, respectively) was used for bleaching and imaging with appropriately adjusted AOTF settings (typically 50 or $100 \%$ for bleaching with the focused laser and 5-10\% for imaging through the spinning disk). Typically 10 prebleach frames were followed by $30-50$ repeated cycles of consecutive bleaching and acquisition steps.

Quantitative evaluation was performed using ImageJ (http://rsb.info.nih.gov/ij/). First, the nuclear area of cells within the bleached region was selected either manually or by threshold-based segmentation. Then the mean fluorescence intensity over time was extracted, background subtracted and normalized to the mean of the last five prebleach values. For one experiment, 20-50 cells in three to five independent time series were investigated per buffer condition. Values obtained in different media conditions were normalized with respect to intensity values in PBS. The mean values of fluorescence intensity of one day of experiments were fitted with a bi-exponential decay function of the form $y=A_{1} \times \exp \left(-x / \tau_{1}\right)+A_{2} \times$ $\exp \left(-x / \tau_{2}\right)$ in Origin 8.0 (OriginLab). Experiments were 
repeated at least twice on different days and mean values and standard deviations of these experiments are given in the supporting information. Values of photobleaching lifetime were calculated according to $\tau_{\mathrm{m}}=A_{1} \times \tau_{1}+A_{2} \times \tau_{2}$ with respect to the value for PBS to correct for differences in bleaching and imaging settings.

\section{Sample and surface preparation for single-molecule measurements}

LabTek 8-well chambered cover slides (Nunc) with a volume of $\sim 750 \mu \mathrm{l}$ were treated with $0.1 \% \mathrm{HF}$ for $30 \mathrm{~s}$ and were washed three times with PBS. Subsequently, they were incubated with a solution of $5 \mathrm{mg} \mathrm{ml}^{-1} \mathrm{BSA}$ and $1 \mathrm{mg} \mathrm{ml}^{-1}$ BSA/biotin in PBS for at least 10 hours at $4{ }^{\circ} \mathrm{C}$ (all compounds were used as received from Sigma Aldrich). After washing, the surface was incubated with a $\sim 0.1 \mathrm{mg} \mathrm{ml}^{-1}$ solution of streptavidin for $5 \mathrm{~min}$ and was washed three times with PBS. Then a biotinylated 40mer oligonucleotide bearing $\mathrm{Cy} 3 \mathrm{~B}$ at position 5 (3'-CGT AT*A GCT ATA CAA TAT AAG TGT AAG GAA TCG AAT CGT A-5' with T* = Cy3B (strand I); as received from IBA, Germany) was incubated on the surface for $1 \mathrm{~min}$ at high concentration $\left(\sim 10^{-8}-10^{-9} \mathrm{~mol} \mathrm{l}^{-1}\right)$. Subsequently, a complementary strand internally labeled with a far-red dye at position 24 (5'-GCA TAT CGA TAC ATT AT*A TTC ACA TTC CTT AGC TTA GCA T-3' with $\mathrm{T}^{*}=\mathrm{ATTO} 647 \mathrm{~N}$ or ATTO 655 (strand II); as received from IBA) was hybridized to strand I yielding a dsDNA probe.

Single-molecule experiments were carried out at room temperature $\left(22 \pm 1{ }^{\circ} \mathrm{C}\right)$ : (i) If not stated otherwise standard PBS pH 7.4 was used. (ii) If indicated, oxygen was removed using an oxygen-scavenging system (PBS, pH 7.4, 10\% (w/v) glucose, $12.5 \%(\mathrm{v} / \mathrm{v})$ glycerine, $50 \mu \mathrm{g} \mathrm{ml}^{-1}$ glucose-oxidase, 100-200 $\mu \mathrm{g} \mathrm{ml}^{-1}$ catalase, and $0.1 \mathrm{mM}$ Tris(2-carboxyethyl)phosphine hydrochloride). (iii) Different concentrations of redox active agents or commercially available AFs were added to the buffer as described in the text and figure captions.

\section{Single-molecule fluorescence spectroscopy}

A custom built confocal microscope was used as described in ref. 19 to study single molecules. The excitation wavelength of a pulsed supercontinuum-source (SuperK Extreme, Koheras) was selected by a combination of an internal and an external acousto-optical tunable filter (external AOTF: AOTFnc-VIS, AA optoelectronic). The excitation wavelength for $\mathrm{Cy} 3 \mathrm{~B}$ was 533, and was set to $640 \mathrm{~nm}$ for ATTO 655 and ATTO $647 \mathrm{~N}$ (spectral width of $2 \mathrm{~nm}$ ). After spatially filtering using a singlemode fiber the beam was coupled into an oil immersion objective $(60 \times$, NA 1.35 , UPLSAPO $60 \mathrm{XO}$, Olympus) of an inverse microscope by a dual-band dichroic beam splitter for recording fluorescent transients (Dualband z532/633, AHF Analysentechnik). Average excitation intensities for all experiments were $4.0 \mathrm{~kW} \mathrm{~cm}$ cm $^{-2}$ at 533 and $1.5 \mathrm{~kW} \mathrm{~cm}^{-2}$ at $640 \mathrm{~nm}$. The resulting fluorescence was collected by the same objective, focused onto a $50 \mu \mathrm{m}$ pinhole, filtered (Brightline HC 582/75 for Cy3B, ET-Bandpass $700 / 75 \mathrm{M}$ for ATTO 655/ATTO 647N, both AHF Analysentechnik), and detected by two avalanche photodiodes (SPCM-AQR-14, PerkinElmer).
Custom made LabVIEW software was used to register the signal and for subsequent data evaluation.

The following procedure was conducted to obtain $\mathrm{ON}$-counts and OFF-times of single-molecule transients: (i) an autocorrelation of the respective fluorescence transients was generated. (ii) the autocorrelation-curve was fitted using an exponential function; (iii) the OFF-times $\tau_{\text {off }}$ and ON-counts $N_{\text {on }}$ were derived from the amplitudes and the characteristic timeconstant of the autocorrelation after background correction according to ref. 19 . On average $\sim 30$ molecules were measured and evaluated for each data point as described for each experimental condition.

\section{Results and discussion}

The four different AFs that we compared in this study are depicted in Fig. 2. Two of the AFs are ROXS based, one of which contains the reductant ascorbic acid (AA) and the oxidant $N, N$-methyl viologen (MV). The ubiquitous oxidant oxygen is removed enzymatically. This buffer system is referred to as ROXS (AA/MV). The second ROXS based AF consists of the reductant Trolox (TX) and uses its quinone derivate Trolox-quinone (TQ) as oxidant in combination with enzymatic oxygen removal and is referred to as ROXS (TX/TQ). The typical concentration of each component (AA/MV/TX) in the respective buffer is $1 \mathrm{mM}$. TQ was shown to be formed in concentrations of $>25 \mu \mathrm{M}$ after dissolving TX in aqueous buffer or under UV-illumination and hence doesn't have to be added to the buffer. . $^{9,10,16}$

Both combinations of ROXS-buffers (AA/MV or TX/TQ) could already prove their suitability to stabilize or alter the fluorescence properties of single fluorophores by photo-induced redox-reactions and are intensively used in single-molecule spectroscopy and super-resolution imaging. $9,10,16,29,30$

The commercial AFs, namely Vectashield (VS) and Ibidi mounting medium (Ibidi-MM) contain phenylenediamine (PPD) and DABCO, respectively. In addition, these mounting media contain between 70 and $90 \%$ glycerol. Thus, they are highly viscous and intrinsically exhibit at least a 3 -fold reduced oxygen concentration compared to water. ${ }^{31}$ This means that the ROXS buffers provide the additional advantage of a lower oxygen concentration in the samples contributing to a more efficient suppression of photobleaching. We believe, however, that the reduced oxygen concentration in glycerol plus the

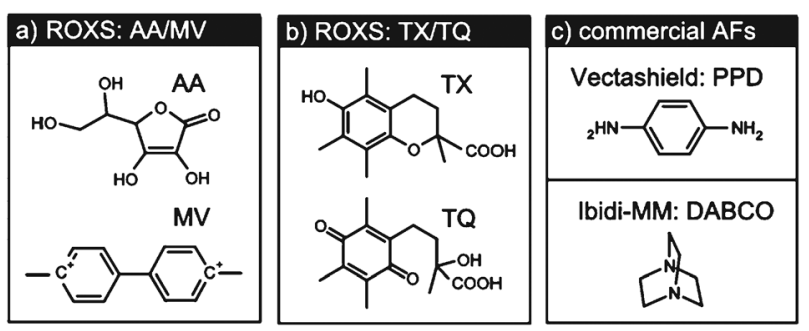

Fig. 2 Chemical structures of the main compounds found in commercial AFs and ROXS-buffers: a) ROXS with the reductant AA and oxidant MV, ROXS (AA/MV); b) ROXS with the reductant TX and oxidant TQ, ROXS (TX/TQ); c) main compound of the commercial AF Vectashield: reductant PPD; main compound of the commercial Ibidi-MM: reductant DABCO. 
reduced mobility lead to comparable conditions for ROXS and commercial AFs as also in samples with enzymatic oxygen removal the final oxygen concentration only reaches the lower micromolar range. ${ }^{32}$

To compare the different AFs for immunofluorescence we performed photobleaching experiments in a commercial confocal microscope frequently used for biological studies (UltraVIEW VoX, PerkinElmer, details see Materials and Methods). Human HeLa cells grown on microscope coverslips were immunolabeled with antibodies conjugated to different fluorophores. Fluorophore-coupled antibodies against GFP were used to label expressed GFP-tagged histone H2B-GFP with an organic dye of choice (Cy3B, ATTO 550, ATTO $647 \mathrm{~N})$. Histone H2B-GFP is stably incorporated into nucleosomes of chromatin and is evenly distributed within the nucleus. Alternatively, histones were detected with primary mouse monoclonal antibodies against Pan-histone and secondary anti-mouse antibodies conjugated to the respective dyes (Alexa 488, Alex 594, Alexa 647). Regions with cells were imaged and subsequently bleached in iterative cycles; the average fluorescence intensity of bleached regions was registered after each cycle.

\section{ATTO 647N as an example for carborhodamine dyes}

Fig. 3 shows exemplary images of typical bleaching experiments with the fluorophore ATTO 647N. This carborhodamine type dye (structure see Scheme 1) is known for its outstanding photophysical performance $\left(\varepsilon_{\max }=1500001 \mathrm{~mol}^{-1} \mathrm{~cm}^{-1}\right.$, $\phi_{\mathrm{fl}}=0.65$ as stated on the ATTO-TEC homepage, www.atto-tec.com) especially on the single-molecule level. ${ }^{33}$

Fluorescent nuclei of cells embedded in either PBS or Vectashield show hardly any remaining fluorescence after 40 bleaching cycles reflecting ensemble bleaching of the fluorophore (Fig. 3a/b). In contrast, when using ROXS (AA/MV) as buffer medium only partial bleaching of ATTO $647 \mathrm{~N}$ is observed (Fig. 3c) indicating a stronger resistance against photobleaching.

For quantitative evaluation the mean fluorescence intensity over time was determined (details see Material and Methods). On average for each buffer condition, 10 independent time series (10-20 nuclei each) were recorded at least on two different days (Fig. 4).

The reference bleaching behaviour of PBS is represented by black squares in Fig. $4 \mathrm{a}$; the intensity is reduced to $\approx 0.25$ of its original value after $\approx 35$ bleaching cycles $(b c)$. The experimental data is well reproduced by a bi-exponential decay (solid lines in Fig. 4a). Results from repetitive experiments and fitting of these data are summarized in Table S1; fit curves from a representative experiment are shown in Fig. 4a together with experimental data. According to these results, the fluorophores bleach with two different bleaching components and a mean bleaching lifetime $\tau_{\mathrm{m}}=23 \pm 2 \mathrm{bc}$ with $\tau_{\mathrm{m}}=A_{1} \times \tau_{1}+A_{2} \times \tau_{2}$. A non-exponential behavior may be attributed to differing microenvironments of the fluorophores and has been reported before. ${ }^{15}$

VS shows a very similar relative bleaching lifetime of $1.3 \pm 0.6$ with respect to PBS but with a considerable amplitude of a fast decay component and overall large experimental errors

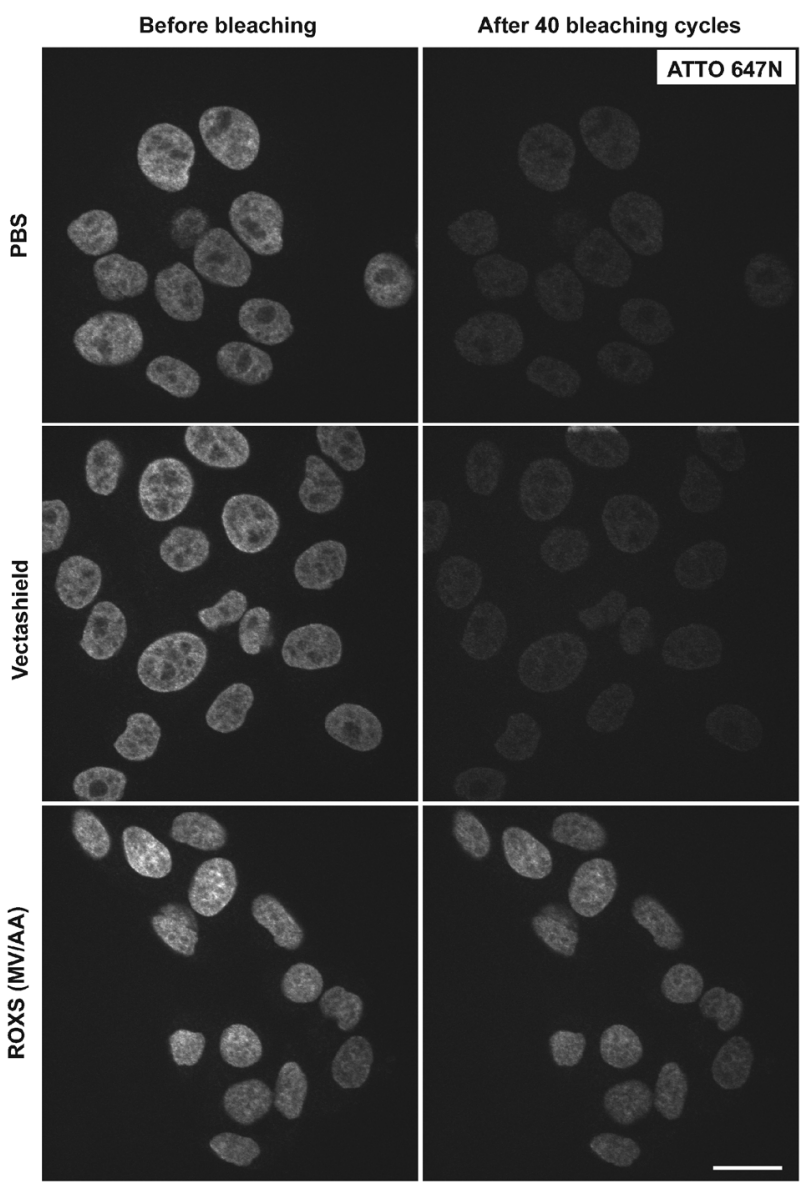

Fig. 3 Confocal images of HeLa histone H2B-GFP expressing cells labeled with ATTO $647 \mathrm{~N}$-coupled GFP antibodies. Samples were embedded using different mounting media. Selected images of a time series with (a) PBS (b) Vectashield and (c) ROXS (AA/MV) are shown here exemplarily. The ATTO $647 \mathrm{~N}$ dye was excited at $635 \mathrm{~nm}$ (close to its absorption maximum at $\approx 644 \mathrm{~nm}$ ). The panels show fluorescently labelled nuclei before and after 40 iterative cycles of confocal imaging and bleaching the entire field of view. Significantly less fluorescence intensity is left in the PBS- and VS-embedded samples, whereas ROXS effectively preserves fluorescence. Bar is $20 \mu \mathrm{m}$.

between different measurements (Fig. 4a, circles; Table S1).§ A significant increase of the photobleaching resistance of ATTO $647 \mathrm{~N}$ is observed for Ibidi-MM, ROXS (AA/MV) and ROXS (TX/TQ) with mean bleaching lifetimes over several experiments of $2.3 \pm 0.2,2.9 \pm 0.5$ and $3.1 \pm 0.5$ relative to PBS, respectively (Table S1). In all cases the fraction of the fast bleaching component is significantly reduced to $\approx 10 \%$ (Fig. 4a). These data quantitatively evidence that both ROXS buffers and Ibidi-MM are able to significantly enhance photostability of ATTO $647 \mathrm{~N}$ in fluorescence imaging.

In the next step we compare the mean brightness of the fluorophores, i.e., of fresh samples (mounted shortly before performing photobleaching experiments) and aged samples (after 3 days storage at $4{ }^{\circ} \mathrm{C}$ ) for the various media

$\S$ Note that all relative bleaching constants in Table S1 and in the text are derived from several measurement days, while the data in the Fig. $4 \mathrm{a} / 5 \mathrm{a} / 6 \mathrm{a} / \mathrm{S} 1 \mathrm{a} / \mathrm{S} 2 / \mathrm{S} 3 \mathrm{a}$ ) show representative mean bleaching curves from a particular experimental day. 
(Fig. 4b). For a systematic comparison we have chosen an intermediate "storage-time" of 3 days to be able to detect significant changes in fluorescence intensities that would allow predictions on the general suitability of the respective medium to preserve fluorescence upon storage. We are convinced that the binding capabilities of antibodies do not play a significant role in our case for the following reasons. (i) The samples were $4 \%$ formaldehyde fixed, a generally accepted way to stably fix and preserve biological structures. (ii) We used approved, highly affine antibodies (see Material and Methods) and we did not observe any unbound fluorescent precipitates or a suspicious increase in background fluorescence after long-term storage that would hint to a dissociation of antibodies from their epitopes. (iii) We know from long-standing experience with similar immunofluorescently-stained samples that they can be stored for many months when either mounted in glycerol-based antifades or in PBS without obvious changes in fluorescence intensities or structure.

While VS and Ibidi-MM reduce the average fluorescence intensity for a freshly prepared solution to $70 \%$ and $40 \%$ relative to $\mathrm{PBS}$, respectively, both ROXS-AFs preserve the brightness of ATTO $647 \mathrm{~N}$. A moderate decrease of ATTO $647 \mathrm{~N}$ brightness was observed after storage in both ROXS media, while storage in VS and PBS did not significantly affect fluorescence intensity. Interestingly, a recovery of mean brightness was observed in Ibidi-MM embedded samples, reaching about the same level as VS.

These data provide valuable information for the fluorophore choice for the desired imaging application considering (i) photobleaching resistance of the fluorophore in the AF medium (Fig. 4a) and quantified by the obtained fitparameters (see below), as well as (ii) fluorescence brightness of the fluorophores in the AF medium and (iii) storage stability of the fluorophores in the AF medium (Fig. 4b).

While the first parameters are obvious quality criteria in fluorescence microscopy many applications might also require storage stability of the sample. In particular for immunofluorescence staining experiments, adherent cells, tissue or whole mount samples are typically embedded in a layer of mounting medium (either hardening or glycerol based and sealed with nail polish) between a microscope slide and a coverslip. Such samples should ideally be able to be stored for days or weeks without major loss of quality.

\section{Rhodamine dyes}

To check for the general validity of the findings for ATTO $647 \mathrm{~N}$ we repeated the measurements for different fluorophores from the dye classes of rhodamines and cyanines, with absorption/ emission spectra ranging from the blue to red spectral range.

For Alexa 488, a fluorophore with absorption/emission in the blue/green region of the visible spectrum, ${ }^{23}$ results from photobleaching experiments are depicted in Fig. 5a.

All experimental decays are again well-described by bi-exponential functions; fit results are shown in Table S1. Surprisingly, we do not observe significant improvement of the photostability for both Ibidi-MM (Fig. 5a, stars; relative $\tau_{\text {mean }}=1.3 \pm 0.2$ ) and $\operatorname{ROXS}(\mathrm{AA} / \mathrm{MV})$ buffer (Fig. 5a, triangles; $\tau_{\text {mean }}=1.1 \pm 0.1$ ) with respect to PBS. While the ROXS (TX/TQ) buffer increases the relative $\tau_{\text {mean }}$ by $1.8 \pm 0.2$ (Fig. 5a, inverted triangles) we observe the best performance for VS with a relative $\tau_{\text {mean }}=2.9 \pm 0.4$ with respect to PBS (Fig. 5a, squares). The fluorescence brightness of Alexa 488 is similar to PBS for all AFs (Fig. 5b). However, storage in ROXS (AA/MV) leads to a substantial loss in fluorescence $(<50 \%)$, while in VS, Ibidi-MM and ROXS (TX/TQ) mounted samples show no significant decrease of brightness.

Other investigated rhodamine dyes were ATTO 550, a fluorophore with absorption and emission in the green/yellow spectral range, and Alexa 594, a fluorophore with excitation and emission maxima in the orange/red spectral range. Both show a similar trend in terms of photostabilizing abilities and fluorophore brightness as reported in the previous sections: for ATTO 550 all AFs significantly increase photobleaching resistance compared to PBS (Fig. S1a, squares and fit; Table S1) by 1.2 for Ibidi-MM, $\approx 2.5$ for VS and $\operatorname{ROXS}(\mathrm{AA} / \mathrm{MV})$ up to a maximum of 3.2 for ROXS (TX/TQ); results are summarized
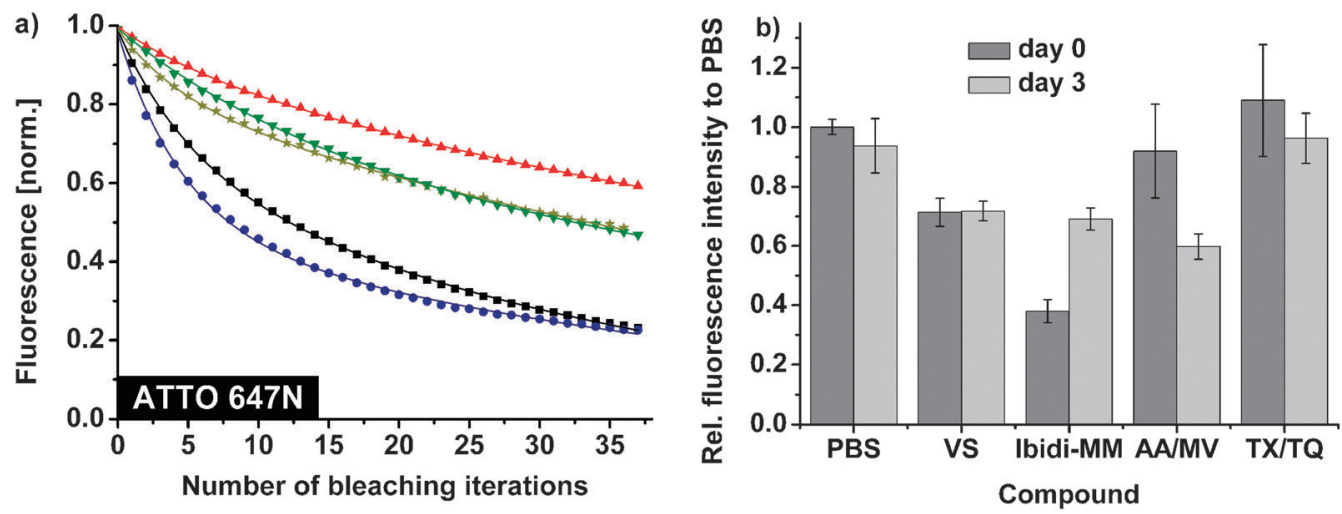

Fig. 4 Results from photobleaching experiments of the dye ATTO 647N obtained from confocal microscopy. (a) Time-course of the normalized fluorescence intensity over 40 bleaching cycles for different AFs: PBS, squares; VS, circles; Ibidi-MM, stars; ROXS (AA/MV), triangles; ROXS (TX/TQ), inverted triangles. Mean curves of at least five measurements from one particular measurement day are shown. Standard deviations were typically below 0.02 and are thus omitted for clarity. Solid lines show a bi-exponential fit with obtained fit-parameters as listed in Table S1. (b) Relative mean preableach fluorescence intensities for fresh samples and for aged samples mounted in different AFs (day $0=$ fresh sample; day $3=$ sample aged for three days at $4{ }^{\circ} \mathrm{C}$ ). Error bars indicate the standard error of mean intensities. 

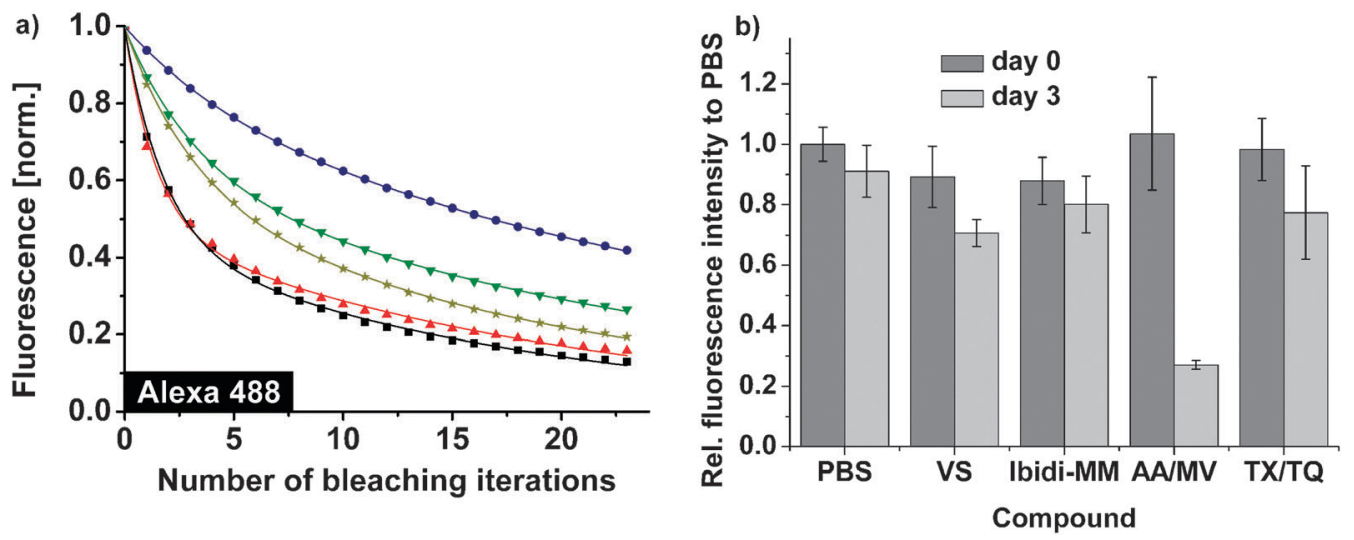

Fig. 5 Photobleaching experiments of the dye Alexa 488. (a) Time-course of the normalized fluorescence intensity over $\approx 25$ bleaching cycles for different buffers: PBS, squares; VS, circles; Ibidi-MM, stars; ROXS (AA/MV), triangles; ROXS (TX/TQ), inverted triangles. Mean curves of at least five measurements from one particular measurement day are shown. Standard deviations were typically below 0.02 and are thus omitted for clarity. Bi-exponential fits are shown as solid lines in the color of the respective data set. Fit results are summarized in Table S1. (b) Relative fluorescence intensity for the different AFs before photobleaching and for aged samples (day $0=$ fresh sample; day $3=$ sample aged for three days). Error bars indicate the standard error of mean intensities.

in Fig. S1a and Table S1. We note a significant enhancement of the brightness of ATTO 550 in Ibidi-MM compared to other AFs. Upon storage in both ROXS media we observe a similar drop in fluorescence as for Alexa 488 (Fig S1b). Experiments with Alexa 594 also follow these described trends: all AFs have a positive influence on the photobleaching resistance of the dye with moderate increases by a factor of $1.5 \pm 0.1$ for ROXS (AA/MV) with respect to PBS (Fig. S2, Table S1). Both VS and ROXS (TX/TQ) show a significant increase by factors of 2.5 and 2.9 , respectively, while Ibidi-MM performs outstandingly well for Alexa 594 with only $15 \%$ fluorescence loss compared to PBS (Fig. S2a, note that the data could not be fitted to a bi-exponential function). Again a moderate increase of brightness is observed for Ibidi-MM and for ROXS (AA/MV) shortly after mounting while the fluorescence intensity seems to be better preserved by VS and Ibidi-MM when storing the sample (Fig. S2b).

\section{Cyanine dyes}

In the next section we investigated the influence of AFs on the photophysical properties of cyanine dyes. As a first example we chose Cy3B, a fluorophore with an absorption- and emissionmaximum at $558 \mathrm{~nm}$ and $572 \mathrm{~nm}$, respectively. This dye is frequently used in single-molecule FRET studies. ${ }^{34,35}$

As already found for rhodamine dyes all AFs clearly increase the resistance of the fluorophore $\mathrm{Cy} 3 \mathrm{~B}$ against photobleaching (Fig. 6a).

The mean bleaching lifetime of PBS (Fig. 6a, squares) is increased by factors between 1.6 and 2.1 for ROXS (AA/MV), Ibidi-MM, VS and ROXS (TX/TQ), compare Fig. 6a and Table S1.

For all AF media we observed a significant decrease in brightness of the fluorophore - a result that is surprising considering that ROXS (AA/MV) was shown to increase the brightness of surface-immobilized or diffusing fluorophores in
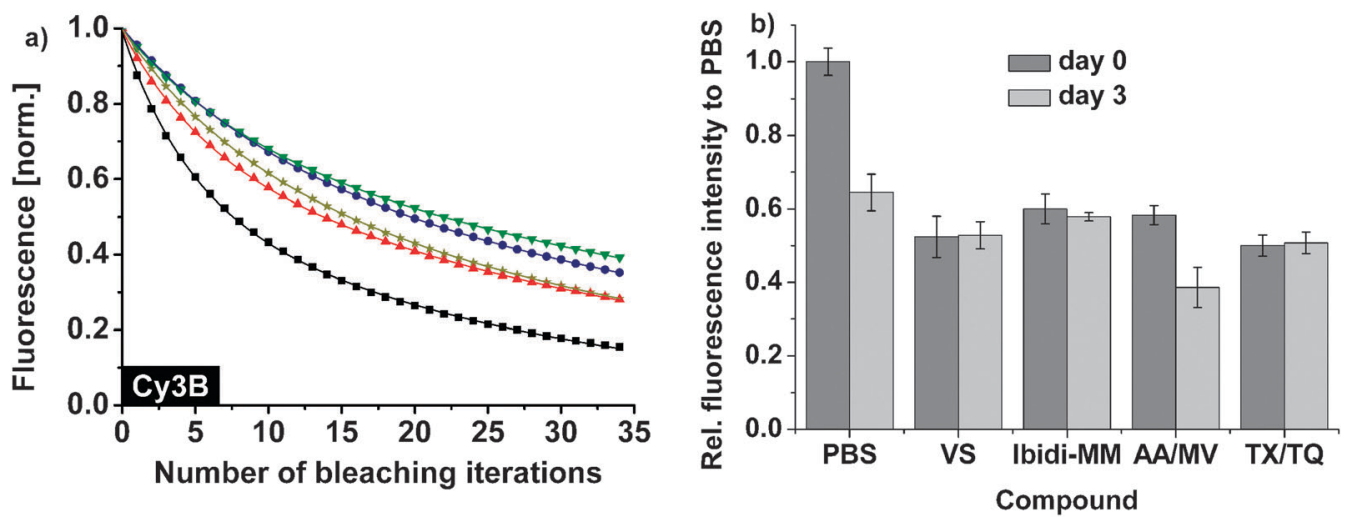

Fig. 6 Photobleaching experiments of the dye Cy3B. (a) The graph shows the time-course of the normalized fluorescence intensity over $\approx 35$ bleaching cycles for different AFs: PBS, squares; VS, circles; Ibidi-MM, stars; ROXS (AA/MV), triangles; ROXS (TX/TQ), inverted triangles. Mean curves of at least five measurements from one particular measurement day are shown. Standard deviations were typically below 0.02 and are thus omitted for clarity. Bi-exponential fits are shown as solid lines in the color of the respective data set. Fit results are summarized in Table S1. (b) Relative fluorescence intensity for the different AFs before photobleaching and for different aging-stages (day $0=$ fresh sample; day 3 = sample aged for three days). Error bars indicate the standard error of mean intensities. 
single-molecule experiments. ${ }^{16}$ The brightness appeared to be relatively stable after storage for all AFs (Fig. 6b).

Another commonly used cyanine dye is Alexa 647, a dye that is very similar to Cy5 regarding its photophysical properties as well as its molecular structure (see Scheme 1 and ref. 25). This dye has an excitation maximum at $650 \mathrm{~nm}$ and shows its emission maximum at $668 \mathrm{~nm}$. Photobleaching experiments reveal interesting findings (Fig. S3a, Table S1): only moderate improvement of the photobleaching resistance is observed for Ibidi-MM (factor of 1.1). The ROXS-buffers improve both photobleaching resistance and fluorophore brightness by factors of $\approx 3$ (Fig. S3, Table S1). In contrast, the fluorophore intensity was substantially reduced to less than $20 \%$ in VS relative to PBS. This results in large experimental errors for the determination of the photobleaching time (data not shown). These facts indicate that this dye is not well suited to be mounted with VS. The dye also shows poor storage stability in all mounting media (Fig. S3b) and is hence better substituted by ATTO 647N.

The presented data of dyes allow the following conclusions: (i) The photostability of the investigated fluorophores generally benefits from the addition of either of the AFs (VS/Ibidi-MM/ROXS) independently from the spectral properties of the dye. (ii) They undergo moderate to substantial increase in their photobleaching resistance with the best average AF-performance of ROXS (TX/TQ), which shows a substantial increase in the photobleaching resistance for all six studied dyes (Table S1). (iii) Fluorophore brightness is typically unaffected or only slightly changed in all AFs compared to PBS alone except for a major decrease observed for Alexa 647 in VS. (iv) Both glycerol-based mounting media, VS and Ibidi-MM, seem better suited for storage of the studied fluorophores compared to ROXS media due to their brightness conserving properties.

\section{Single-molecule sensing of redox properties of commercial AFs}

The quantitative comparison of AF characteristics in their application using fluorescently labelled biological samples revealed preferences for different dyes and quite specific interactions such as the fluorescence loss of Alexa 647 when used with VS. Whereas the ROXS AFs function is described by a photophysical model, DABCO (main component in Ibidi-MM) and PPD (main component in VS) were selected empirically. To address the underlying photophysical and physico-chemical mechanisms and to elucidate whether a ROXS-like mechanism might also be involved with these empirically found AFs, we carried out single-molecule measurements with the SMRS ATTO 655. We therefore recorded single-molecule transients of ATTO 655 labelled DNA, immobilized via biotin/streptavidin on BSA surfaces and analyzed the blinking kinetics of ATTO 655 at different AF concentrations. It has been shown before, ${ }^{10,19}$ that the number of photons emitted during one ON-period $\mathrm{N}_{\text {on }}$ is an inverse measure for the reducing properties of the environment and that the OFF-times $\tau_{\text {off }}$ are a measure of the oxidizing properties. Increasing concentrations of AA, for example, at constant concentrations of the oxidant $\mathrm{MV}$, reduce the number of $\mathrm{N}_{\text {on }}$ since the fluorophore is transferred to its radical anion

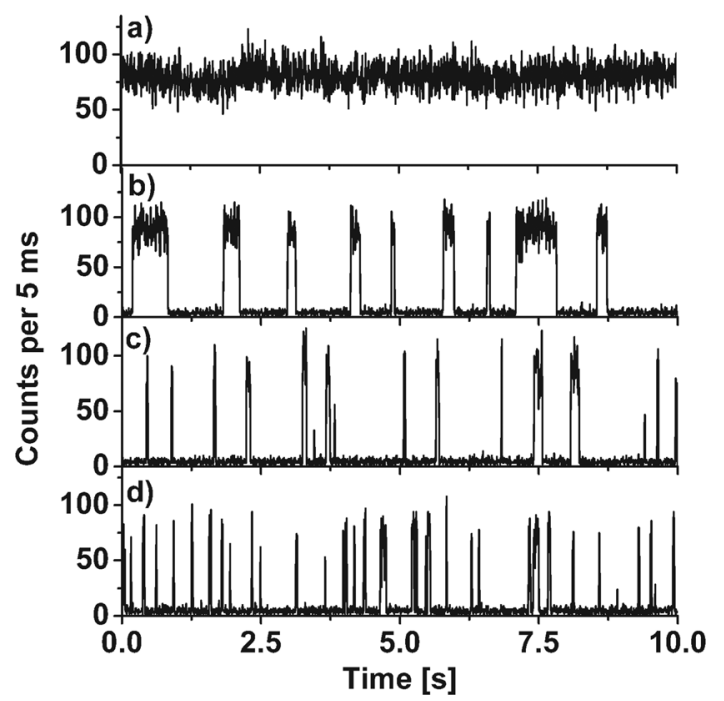

Fig. 7 Fluorescence transients of the single-molecule redox sensor ATTO 655 in PBS and at varying concentrations of VS (details see Materials and Methods): (a) PBS, (b) PBS with $0.07 \%$ v/v VS-stock solution (SMRS-values: $\tau_{\text {on }}=450 \pm 190 \mathrm{~ms}, \tau_{\text {off }}=990 \pm 560 \mathrm{~ms}$; derived from on/off histograms), (c) PBS with $0.22 \% \mathrm{v} / \mathrm{v}$ VS-stock solution (SMRS-values: $\tau_{\mathrm{on}}=75 \pm 9 \mathrm{~ms}, \tau_{\mathrm{off}}=625 \pm 155 \mathrm{~ms}$; derived from on/off histograms), (d) PBS with $0.70 \% \mathrm{v} / \mathrm{v}$ VS-stock solution (SMRS-values: $\tau_{\text {on }}=30 \pm 4 \mathrm{~ms}, \tau_{\text {off }}=390 \pm 150 \mathrm{~ms}$; derived from autocorrelation analysis).

state more rapidly. Increasing concentrations of MV, on the other hand, reduce $\tau_{\text {off. }}{ }^{19}$

Fig. 7 shows representative fluorescence transients of surface-immobilized ATTO 655 molecules in PBS (Fig. 7a) and with different concentrations of VS-medium (Fig. 7b-e). The fluorescence of ATTO 655 in PBS in the absence of redoxactive agents is comparatively stable with rare OFF-states and no amplitude in the autocorrelation function down to 1 $\mu$ s (not shown).

Adding VS to the solution at a concentration of $0.07 \% \mathrm{v} / \mathrm{v}$ results in the appearance of blinking with average values of $\tau_{\text {on }}=450 \pm 190 \mathrm{~ms}$ and $\tau_{\text {off }}=990 \pm 560 \mathrm{~ms}$ (Fig. $7 \mathrm{~b}$ shows a representative transient; sensor values were derived from on/off histograms). Since the excitation intensity was the same for all measurements, $\tau_{\text {on }}$ is proportional to $\mathrm{N}_{\text {on }}$ and is used in our discussion. The OFF-state is attributed to a reduction reaction into a metastable radical anion. Since oxygen that also acts as an oxidant is not removed in these measurements and because $0.07 \%$ VS does not alter the oxygen solubility and diffusion constant significantly, the value of $990 \mathrm{~ms}$ has to be compared to the literature value of $\tau_{\text {off }}=(1800 \pm 1000) \mathrm{ms}$ for PBS with AA. ${ }^{19}$ Increasing the concentration of VS to $0.22 \%$ $\mathrm{v} / \mathrm{v}$ changes both values significantly to $\tau_{\text {on }}=75 \pm 9 \mathrm{~ms}$ and $\tau_{\text {off }}=625 \pm 155 \mathrm{~ms}$ (Fig. 7c shows a representative trace; average sensor values derived from on/off histograms). Further increase to $0.70 \% \mathrm{v} / \mathrm{v}$ VS-stock solution changes the sensor values to $\tau_{\text {on }}=30 \pm 4 \mathrm{~ms}, \tau_{\text {off }}=390 \pm 150 \mathrm{~ms}$ (Fig. 7d; sensor values derived from autocorrelation analysis of intensity transients). The 15 -fold reduction of $\tau_{\text {on }}$ reflects the known reducing properties of PPD. The simultaneous 3 -fold reduction of $\tau_{\text {off }}$ indicates the presence of some oxidizing properties of the AF medium. The decrease of $\tau_{\text {off }}$ is certainly 


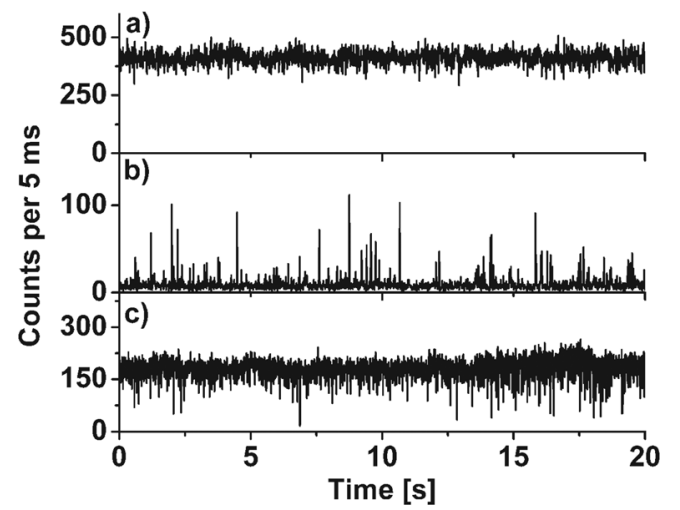

Fig. 8 Fluorescence transients of single ATTO $647 \mathrm{~N}$ molecules in (a) ROXS buffer ( $1 \mathrm{mM} \mathrm{AA/MV}$, enzymatic oxygen removal and (b) $0.14 \% \mathrm{v} / \mathrm{v}$ VS-stock solution dissolved PBS with enzymatic oxygen removal and (c) pure VS-stock solution.

significant and might be weakened by reduced oxygen solubility and mobility at higher VS fractions. The amount of oxidant in the solution is substantially smaller than that of the reductant and may hence be attributed to oxidizing impurities or chemical products of PPD upon dissolving in buffer or glycerol.

These results and their interpretation are supported by similar experiments with the dye ATTO 647N: single, immobilized molecules show extremely stable fluorescence in ROXS (AA/MV) as well as in ROXS (TX/TQ); Fig. 8a). The addition of small amounts of VS to a deoxygenated PBS solution (Fig. 8b) does not increase photostability in a similar manner; here strong blinking of ATTO $647 \mathrm{~N}$ is observed with short ON-times and long OFF-periods (Fig. 8b) similar to observation in the absence of reductant due to triplet blinking or in the presence of only reductants due to radical ion formation. Replacing PBS by pure VS, however, already shows drastic reduction of blinking and allows ATTO $647 \mathrm{~N}$ to fluoresce for a longer time-period (Fig. 8c).

These results are in accordance with the expectations of the ROXS concept. In order to fluoresce stably, the dye ATTO $647 \mathrm{~N}$ needs a sufficient amount of both oxidant and reductant. For low concentration of VS the need for an oxidant is not fulfilled as the concentration is too low, while at higher concentration both redox-compounds are present in sufficient concentrations.

Next, we carried out analogous measurements for Ibidi-MM (Fig. 9). We also find a reduction of both sensor values upon increasing concentration of Ibidi-MM pointing to similar properties of this DABCO containing medium compared to VS.

Quantitatively, however, the properties of Ibidi-MM are quite different to VS since $\tau_{\text {on }}$ only changes 3.7 -fold whereas $\tau_{\text {off }}$ is reduced 15 -fold. These data indicate that Ibidi-MM has substantial oxidizing properties and comparatively mild reducing properties.

The results presented in the current section clearly show that VS and Ibidi-MM might have been selected because in the buffer applied they exhibit both reducing and oxidizing properties and thus imply some aspects of the ROXS concept. At this point we do not know the origin of the reducing and oxidizing properties and can only speculate whether the formation of oxidizing species is related to partial degradation

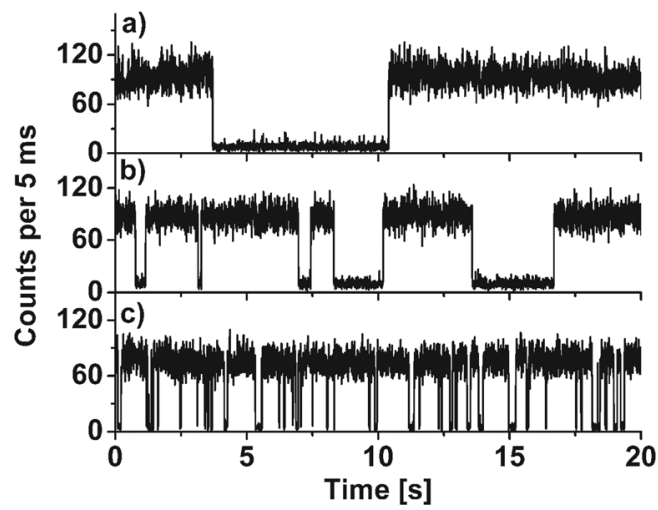

Fig. 9 Fluorescence transients of ATTO 655 in PBS for varying concentrations of Ibidi-MM: a) PBS with $0.1 \% \mathrm{v} / \mathrm{v}$ Ibidi-MM-stock solution (SMRS-values: $\tau_{\text {on }}=2200 \pm 1100 \mathrm{~ms}, \tau_{\text {off }}=1100 \pm 600 \mathrm{~ms}$ ), (b) PBS with $7 \% \mathrm{v} / \mathrm{v}$ Ibidi-MM-stock solution (SMRS-values: $\tau_{\text {on }}=1600 \pm 700 \mathrm{~ms}, \tau_{\text {off }}=710 \pm 200 \mathrm{~ms}$ ), (c) PBS with $33 \%$ $\mathrm{v} / \mathrm{v}$ Ibidi-MM-stock solution (SMRS-values: $\tau_{\text {on }}=600 \pm 170 \mathrm{~ms}$, $\left.\tau_{\text {off }}=75 \pm 40 \mathrm{~ms}\right)$. All SMRS values were derived from on/off-histograms of single-molecules $(\approx 30-40)$.

of the original reducing compound. As mentioned above, such a mechanism has been demonstrated for Trolox yielding Trolox-quinone. ${ }^{10}$ Our investigations hence show that the principle of triplet-state quenching by redox-active agents and recovery of the fluorophore's ionized states by subsequent redox-reactions (the ROXS concept, see Fig. 1) is of fundamental importance to prevent photobleaching in all fluorescence applications. Our study also reveals that, though the chemical and physical reasons for the successful application of several AFs were unknown, their working action can be traced back to a combination of oxidizing and reducing agents.

\section{Conclusions: which Antifade for which fluorophore?}

In this paper we compared the performance of commonly used and commercially available antifades such as Vectashield and Ibidi-MM with new AFs based on the recently developed ROXS concept. We employed an application-oriented assay using confocal imaging and bleaching of fluorescently labeled cells, and complemented it by single-molecule spectroscopy. We found that generally all AFs exhibit a significant photostabilizing effect. It turned out that no optimal AF could be identified but that different AFs are preferential for different fluorophores and different conditions. Our single-molecule study revealed that this can at least in part be explained by the fact that the commercial AFs Vectashield und Ibidi-MM also exhibit reducing and oxidizing properties and thus use principles of the ROXS concept. These results clearly demonstrate the importance of the ROXS concept for fluorescence applications in general and also show how the empirical development of commercial antifading agents might have lead to the use of compounds that have both reducing and oxidizing capabilities.

The differences between the tested AFs might be related to the different concentrations, mobility and reactivity of the involved redox agents on the one hand as well as to more 
specific interactions between certain $\mathrm{AFs}$ and fluorophores that in some cases even lead to near complete quenching of a fluorophore. A generalized overview with the aim of providing practical guidance to microscopists also including further observations that could not be discussed in detail in the context of this work is provided in the following.

Vectashield performs best with the green emitting dye Alexa 488 (widely used for multicolour applications), performs well with dyes in the orange-red emission range (Cy3b, ATTO 555, Alexa 594) and is compatible with imaging of cyan emitting dyes, such as the DNA binding DAPI and Hoechst (data not shown). In contrast, the DABCO containing Ibidi-MM performs significantly better with far-red emitting dyes and similar or slightly worse than VS in combination with most other dyes. Thus, it offers a good compromise in multicolour application of improved photostability throughout the entire spectrum. The relative high diffraction index (1.440) due to their glycerol-based formulae allows deeper imaging into the sample when using high NA oil objectives. Both commercial AFs provide good storage stability for routine use with typical biological samples.

The ROXS based formulae show comparable or slightly better antifading performance with orange and red emitting dyes and they clearly outperform both glycerol-based media with far-red emitting dyes (Alexa 647 and ATTO 647N). On the other hand, they show only little to moderate effect with green emitting Alexa 488. This indicates that the multi-photon processes become more and more influential with decreasing excitation wavelength. For example in the case of Alexa 488 and ATTO 550, ROXS seems to work less effective compared to red-absorbing dyes, a fact that points to an increasing influence of higher-excited states that contribute to the overall bleaching next to the triplet-route, which is effectively suppressed by ROXS. This interpretation has also been corroborated by other studies exciting, for example, red dyes simultaneously with blue and red light. ${ }^{33,36,37}$

In the direct comparison of redox active agents, TX/TQ appeared in most cases superior to AA/MV in terms of antifading efficiency and storage stability. The antifading performance of ROXS-AFs is, strongly dependent on the oxygen-free environment in aqueous solution, which requires extra sealing of the embedded sample, e.g., with silicone. Storage may be compromised by evaporation and the performance is less reproducible due to variable degrees of oxygen depletion. The lower refractive index of the aqueous solution may reduce image quality due to the refractive index mismatch with the immersion oil. This should be considered in particular when imaging deeper $(>10 \mu \mathrm{m})$ into the sample using high NA oil objectives, whereas near-field applications such as TIRF (often used in single molecule applications, e.g. single-molecule tracking or single molecule localization microscopy) are not compromised. For wide-field applications the usage of waterimmersion objectives may be advantageous with these AFs. Recently, 2,2'-thiodiethanol (TDE) embedding was reported to be superior for stimulated emission depletion (STED) microscopy of ATTO $647 \mathrm{~N}$ labeled samples by having refractive index of 1.52, equal to immersion oil. ${ }^{38}$ In our hands, however, cells embedded in TDE show a flattened morphology similar to the hardening media. While orange and red emitting dyes (e.g., Alexa 555, ATTO 647N) show enhanced fluorescence, other dyes in the lower spectral range (e.g., DAPI, Alexa 488) show strongly decreased brightness in TDE (data not shown).

All tests in this study have been performed on organic dyes conjugated to antibodies. It should be noted that all analyzed AFs had no significant influence on photostability of green fluorescent protein (GFP) when excited with the $488 \mathrm{~nm}$ laser line (data not shown). This finding might be related to the fact that the chromophore formed by the tripeptide Ser65-Tyr66-Gly67 is located protected within the beta-barrel structure of the protein. ${ }^{39}$

Bright and photostable fluorescence reduces exposure-time and increases the signal-to-noise ratio, and is therefore an important prerequisite for high quality imaging of biological samples. This is even more true for applications of recent super-resolution imaging methods, such as single-molecule localization microscopy (e.g., STORM, PALM) ${ }^{7,33}$ STED $^{6}$ and 3-dimensional structured illumination microscopy (3D-SIM) ${ }^{40}$ that are generally more demanding for the employed dyes. The choice of the right antifade medium makes an important contribution to the dye performances. This paper gives useful guidelines for their application and provides a framework for further optimization of antifading formulae of mounting media on the basis of the ROXS principle.

\section{Acknowledgements}

The authors thank C. Eggeling for discussions and helpful information. We thank U. Rothbauer (ChromoTek GmbH) for providing fluorophore coupled GFP antibodies. We are grateful to $\mathrm{S}$. Hölzl for technical assistance. We are indebted to H. Leonhardt for generous support. T. Cordes is supported by a Marie-Curie Intra-European Fellowship provided by the European Commission under the Seventh Framework Programme (grant PIEF-GA-2009-255075). The project was supported by the DFG (Inst 86/1051-1 to P. Tinnefeld and SFB TR5 to L. Schermelleh), the Nanosystems Initiative Munich (NIM) and the BioImaging Network Munich.

\section{References}

1 B. N. G. Giepmans, S. R. Adams, M. H. Ellisman and R. Y. Tsien, Science, 2006, 312, 217-224.

2 W. E. Moerner and L. Kador, Phys. Rev. Lett., 1989, 62, $2535-2538$.

3 M. Orrit and J. Bernard, Phys. Rev. Lett., 1990, 65, 2716-2719.

4 E. Betzig, G. H. Patterson, R. Sougrat, O. W. Lindwasser, S. Olenych, J. S. Bonifacino, M. W. Davidson, J. LippincottSchwartz and H. F. Hess, Science, 2006, 313, 1642-1645.

5 M. J. Rust, M. Bates and X. Zhuang, Nat. Methods, 2006, 3, 793-795.

6 S. W. Hell, Science, 2007, 316, 1153-1158.

7 L. Schermelleh, R. Heintzmann and H. Leonhardt, J. Cell Biol., 2010, 190, 165-175.

8 M. Fernandez-Suarez and A. Y. Ting, Nat. Rev. Mol. Cell Biol., 2008, 9, 929-943.

9 I. Rasnik, S. A. McKinney and T. Ha, Nat. Methods, 2006, 3, 891-893.

10 T. Cordes, J. Vogelsang and P. Tinnefeld, J. Am. Chem. Soc., 2009, 131, 5018.

11 A. N. Fletcher and M. E. Pietrak, Appl. Phys. B: Photophys. Laser Chem., 1985, 37, 151-157.

12 J. Widengren, A. Chmyrov, C. Eggeling, P. A. Lofdahl and C. A. M. Seidel, J. Phys. Chem. A, 2007, 111, 429-440. 
13 R. Dave, D. S. Terry, J. B. Munro and S. C. Blanchard, Biophys. J., 2009, 96, 2371-2381.

14 A. Longin, C. Souchier, M. Ffrench and P. A. Bryon, Journal of Histochemistry \& Cytochemistry, 1993, 41, 1833-1840.

15 M. Ono, T. Murakami, A. Kudo, M. Isshiki, H. Sawada and A. Segawa, Journal of Histochemistry \& Cytochemistry, 2001, 49, 305-311.

16 J. Vogelsang, R. Kasper, C. Steinhauer, B. Person, M. Heilemann, M. Sauer and P. Tinnefeld, Angew. Chem., Int. Ed., 2008, 47, 5465-5469.

17 T. Cordes, J. Vogelsang, M. Anaya, C. Spagnuolo, A. Gietl, W. Summerer, A. Herrmann, K. Mullen and P. Tinnefeld, J. Am. Chem. Soc., 2010, 132, 2404-2409.

18 T. Cordes, I. H. Stein, C. Forthmann, C. Steinhauer, M. Walz, W. Summerer, B. Person, J. Vogelsang and P. Tinnefeld, Proc. SPIE, 2009, 7367, 73671D.

19 J. Vogelsang, T. Cordes, C. Forthmann, C. Steinhauer and P. Tinnefeld, Proc. Natl. Acad. Sci. U. S. A., 2009, 106, 8107-8112.

20 C. Steinhauer, C. Forthmann, J. Vogelsang and P. Tinnefeld, J. Am. Chem. Soc., 2008, 130, 16840.

21 J. Vogelsang, T. Cordes, C. Forthmann, C. Steinhauer and P. Tinnefeld, Nano Lett., 2010, 10, 672-679.

22 C. Eggeling, C. Ringemann, R. Medda, G. Schwarzmann, K. Sandhoff, S. Polyakova, V. N. Belov, B. Hein, C. von Middendorff, A. Schonle and S. W. Hell, Nature, 2009, 457, 1159-U1121.

23 N. Panchuk-Voloshina, R. P. Haugland, J. Bishop-Stewart, M. K. Bhalgat, P. J. Millard, F. Mao, W. Y. Leung and R. P. Haugland, J. Histochem. Cytochem., 1999, 47, 1179-1188.

24 M. Cooper, A. Ebner, M. Briggs, M. Burrows, N. Gardner, R. Richardson and R. West, J. Fluoresc., 2004, 14, 145-150.

25 S. S. White, H. T. Li, R. J. Marsh, J. D. Piper, N. D. Leonczek, N. Nicolaou, A. J. Bain, L. M. Ying and D. Klenerman, J. Am. Chem. Soc., 2006, 128, 11423-11432.
26 U. Rothbauer, K. Zolghadr, S. Tillib, D. Nowak, L. Schermelleh, A. Gahl, N. Backmann, K. Conrath, S. Muyldermans, M. C. Cardoso and H. Leonhardt, Nat. Methods, 2006, 3, 887-889.

27 S. C. Blanchard, R. L. Gonzalez, H. D. Kim, S. Chu and J. D. Puglisi, Nat. Struct. Mol. Biol., 2004, 11, 1008-1014.

28 S. C. Blanchard, H. D. Kim, R. L. Gonzalez, Jr., J. D. Puglisi and S. Chu, Proc. Natl. Acad. Sci. U. S. A., 2004, 101, 12893-12898.

29 A. Pertsinidis, Y. X. Zhang and S. Chu, Nature, 2010, 466, 647-U611.

30 S. V. Solomatin, M. Greenfeld, S. Chu and D. Herschlag, Nature, 2010, 463, 681-U117.

31 M. A. Joslyn and H. Supplee, Food Research, 1949, 14, 209-215.

32 C. E. Aitken, R. A. Marshall and J. D. Pulglisi, Biophys. J., 2008, 94, 1826-1835.

33 J. Vogelsang, C. Steinhauer, C. Forthmann, I. H. Stein, B. Person-Skegro, T. Cordes and P. Tinnefeld, ChemPhysChem, 2010, 11, 2475-2490.

34 J. Vogelsang, S. Doose, M. Sauer and P. Tinnefeld, Anal. Chem., 2007, 79, 7367-7375.

35 Y. Santoso, C. M. Joyce, O. Potapova, L. Le Reste, J. Hohlbein, J. P. Torella, N. D. F. Grindley and A. N. Kapanidis, Proc. Natl. Acad. Sci. U. S. A., 2010, 107, 715-720.

36 C. Eggeling, J. Widengren, L. Brand, J. Schaffer, S. Felekyan and C. A. Seidel, J. Phys. Chem. A, 2006, 110, 2979-2995.

37 X. X. Kong, E. Nir, K. Hamadani and S. Weiss, J. Am. Chem. Soc., 2007, 129, 4643-4654.

38 T. Staudt, M. C. Lang, R. Medda, J. Engelhardt and S. W. Hell, Microsc. Res. Tech., 2007, 70, 1-9.

39 R. Y. Tsien, Аnпи. Rev. Biochem., 1998, 67, 509-544.

40 L. Schermelleh, P. M. Carlton, S. Haase, L. Shao, L. Winoto, P. Kner, B. Burke, M. C. Cardoso, D. A. Agard, M. G. Gustafsson, H. Leonhardt and J. W. Sedat, Science, 2008, 320, 1332-1336. 